\title{
Composition corporelle de poulets cou nu ou normalement emplumés : résultats dans un croisement de type chair
}

\author{
A. EL-ATTAR (*) et P. MERAT \\ I.N.R.A., Laboratoire de Génétique factorielle \\ Centre National de Recherches Zootechniques, F 78350 Jouven-Josas
}

\begin{abstract}
Résumé
Des résultats antérieurs sur des populations légères ou mi-lourdes avaient montré un effet favorable du gène cou nu sur le rendement à l'abattage et le rendement en viande des poulets. Nous avons voulu les vérifier sur un croisement plus lourd de type chair. Des coqs Cornish ont été accouplés à des poules $\mathrm{Nana}$ hétérozygotes pour le gène "cou nu 》 $(\mathrm{Na})$, la moitié de la descendance ayant un plumage normal $(n a+n a+)$, l'autre moitié étant hétérozygote $\mathrm{Na} n \mathrm{na}^{+}$. A l'abattage (60 jours), il n'y a pas de différence de croissance entre les 2 génotypes. Les poulets cou nu des 2 sexes ont une proportion plus faible de plumage par rapport au poids vif (-1,6 p. 100 environ), un meilleur rendement en viande de la carcasse éviscérée (entre 1 et 1,5 p. 100 dans l'ensemble). Cette différence se localise dans la région thoracique. Accessoirement, on note une importance relative un peu plus grande des viscères et du cour et plus faible de la région du cou chez les oiseaux cou nu. Pour l'essentiel, ces résultats sont de même sens que ceux obtenus auparavant, l'importance des différences étant moindre que dans des croisements plus légers si on l'exprime en p. 100 du poids vif mais du même ordre en valeur absolue.
\end{abstract}

Mots clés : Poulet de chair, composition corporelle, gène, cou nu.

\begin{abstract}
Summary
Body composition of «naked neck» or normally feathered chicks : results in a broiler-type cross
\end{abstract}

Previous results ou light or medium-heavy populations had shown a favorable effect of the naked neck gene on dressed carcass percentage and meat yield of chicks. The aim of this work is to verify these results on a heavier broiler-type cross. Cornish males were pedigree mated to $\mathrm{Na} \mathrm{na}+$ females heterozygous for the naked neck ( $\mathrm{Na}$ ) gene, half of the progeny having a normal plumage $(n a+n a+)$, the other half being heterozygous $\mathrm{Na} n a+$. At slaughter age $(60 \mathrm{~d})$, there was no difference in growth rate between the 2 genotypes. Naked neck chicks of both sexes had a lower proportion of plumage in p. 100 of live weight (about -1.6 p. 100), a better meat yield of the eviscerated carcass (between 1 and 1.5 p. 100 on the

* Adresse permanente : Département de Production Animale, Faculté d'Agriculture, Université Aïn-Shams, Le Caire, Egypte. 
whole). This difference was located in the thoracic region. Secondarily, a slightly higher relative importance of viscera and heart and a lower importance of the neck region in naked neck birds are noted. The results are in the same direction as those obtained earlier, the magnitude of the differences being less than in lighter crosses when expressed in p. 100 of live weight but of the same order in absolute terms.

Key words : Broilers, body composition, nacked neck, gene.

\section{Introduction}

Deux travaux antérieurs (ZeIN-EL-Dein et al., 1981 ; ZeIN-EL-Dein et al., 1984 b) ont montré que, outre leur plumage réduit, des poulets cou nu hétérozygotes présentaient dans les 2 sexes une proportion plus élevée de muscles par rapport au squelette et un meilleur rendement en viande de la carcasse éviscérée que des poulets normalement emplumés. Cependant, le premier résultat était obtenu sur des croisements de la race Fayoumi de petite taille, et le second sur une population milourde dont le poids à l'abattage était nettement inférieur à un type poulet de chair standard. Nous avons donc voulu vérifier ces résultats dans un croisement se rapprochant de ce type. Nous avons voulu également localiser les régions corporelles responsables de l'avantage associé au gène cou nu pour le rendement en viande.

\section{Matériel et méthodes}

\section{A. Animaux}

Huit coqs Cornish provenant de l'institut de sélection animale, âgés de 18 mois, ont été accouplés chacun à 5 poules d'une lignée expérimentale au laboratoire de génétique factorielle. Ces poules étaient hétérozygotes $\left(\mathrm{Nana^{+ } )}\right.$ pour le gène cou $n u(N a)$, de sorte que la moitié environ de leur descendance avait le génotype $\mathrm{Na} \mathrm{na}{ }^{+}(\mathrm{cou} n u)$, la moitié nat nat (plumage normal). Deux éclosions semi-pedigree (identification du père seul) avaient lieu, la première le 18-11-1983, la seconde le 6-1-1984. Pour chacune les œufs étaient récoltés sur 3 semaines. Les nombres de poussins éclos viables étaient respectivement 231 pour la première et 205 pour la seconde.

\section{B. Conditions d'élevage}

Chacune des 2 éclosions était élevée dans une loge de poussinière à l'Ecole Nationale d'Aviculture de Rambouillet. L'aliment, distribué ad libitum, était à 21,9 p. 100 de protéines totales et $2930 \mathrm{Kcal} \mathrm{EM} / \mathrm{kg}$ jusqu'à 4 semianes, puis à 20,3 p. 100 de protéines totales et $3060 \mathrm{Kcal} \mathrm{EM} / \mathrm{kg}$. Un chauffage par radiant était assuré jusqu'à 4 semaines. Les animaux étaient éclairés $24 \mathrm{~h}$ /jour. La densité était de l'ordre de 10 animaux au $\mathrm{m}^{2}$.

\section{Mesures}

Les animaux étaient pesés individuellement à $4,6,7$ et 8 semaines pour les 2 éclosions, et avant abattage pour la première éclosion. 
A l'abattage (60 jours d'âge), sur un échantillon au hasard de chaque génotype et sexe dans la première éclosion, le sang était pesé par différence après saignée, ainsi que les plumes après plumaison à sec, puis des mesures de composition corporelle, dont la liste est contenue dans les tableaux 2 et 3, étaient faites à Jouy-en-Josas. Dans la seconde éclosion, pour des raisons matérielles, seul un échantillon de mâles a été mesuré de façon semblable, dans chaque génotype. Les mesures anatomiques appellent les précisions suivantes : la graisse abdominale était prélevée autour du gésier, de la bourse de Fabricius et sur la paroi abdominale et n'incluait pas celle attachée à l'intestin (RICARD \& RouviER, 1967). Sur la demi-carcasse éviscérée, la peau, l'ensemble du squelette et le total de la viande étaient séparés et pesés, ainsi que le gras séparable visuellement, localisé entre les faisceaux musculaires et autour des vaisseaux, auquel était ajoutée la graisse sous-cutanée. La même découpe était réalisée pour les 2 parties de la carcasse constituées par le tronc et le membre inférieur (cuisse et pilon).

\section{Analyses statistiques}

Pour le poids à 8 semaines, seul mentionné ici, une analyse de variance tient compte des facteurs : éclosion, sexe et génotype.

Pour les variables anatomiques, seuls les mâles étant mesurés dans la seconde éclosion, une analyse séparée pour le premier lot inclut les facteurs sexe et génotype; une autre analyse pour le second lot comporte le génotype comme unique source de variation. Une analyse complémentaire sur les mâles des 2 éclosions fait intervenir les facteurs : éclosion et génotype.

\section{Résultats et discussion}

\section{A. Données d'élevage}

Ces données obtenues à l'Ecole Nationale d'Aviculture, sont examinées plus en détail séparément. La mortalité était relativement faible $(9$ morts au total dans la première éclosion, 7 dans la seconde, soit respectivement 3,9 et 3,4 p. 100) et était répartie dans tous les groupes.

Le tableau 1 présente les poids moyens à 8 semaines par sexe, génotype et éclosion. Dans l'ensemble, la croissance jusqu'à cet âge ne diffère pas significativement selon le génotype, cou nu ou plumage normal. Ceci est en accord avec des résultats antérieurs obtenus avec des populations à croissance moins rapide et des conditions voisines de température (BoRdas et al., 1978; MONNET et al., 1979).

\section{B. Variables anatomiques}

A partir des résultats des tableaux 2,3 et 4 , nous ne mentionnerons que pour mémoire les différences de composition corporelle selon l'éclosion qui sont vraisemblablement une conséquence de la croissance moyenne moins rapide pour la seconde éclosion que pour la première, pour des raisons non identifiées. De même, l'interaction significative entre génotype et éclosion pour le pourcentage de viscères et du foie 
est difficile à interpréter. D'autre part, les effets associés au sexe (mâles ayant relativement moins de plumes et de viscères, une proportion plus grande représentée par la tête, le cou, les pattes, le squelette du tronc) sont connus. Quant aux interactions génotype $\times$ sexe significatives dans la première éclosion (tabl. 2) pour le pourcentage du foie et celui de la peau dans la carcasse éviscérée et la région du tronc, elles n'ont pas d'interprétation simple.

\section{TABleau 1}

Poids moyens à 8 semaines par sexe, génotype et éclosion et analyse de variance.

Mean 8 weeks body weight per sex, genotype and hatch and variance analysis.

\begin{tabular}{|c|c|c|c|c|c|c|}
\hline \multicolumn{7}{|c|}{ Valeurs moyennes (g) } \\
\hline & \multicolumn{2}{|c|}{ Mâles } & \multicolumn{2}{|c|}{ Femelles } & \multicolumn{2}{|c|}{ Total } \\
\hline & $\mathrm{Nana}+$ & $n a+n a+$ & $\mathrm{Nana+}$ & $n a+n a+$ & $\mathrm{Nana}+$ & $n a+n a+$ \\
\hline Eclosion $1 \ldots \ldots$ & 2093 & 1994 & 1719 & 1757 & 1906 & 1875 \\
\hline Eclosion $2 \ldots \ldots$ & 1956 & 1938 & 1636 & 1626 & 1796 & 1782 \\
\hline \multicolumn{7}{|c|}{$\begin{array}{c}\text { Analyse de variance : } \\
\text { Valeurs } F \text { et signification par source de variation }\end{array}$} \\
\hline Eclosion & \multicolumn{2}{|c|}{ Génotype } & \multicolumn{2}{|c|}{ Sexe } & \multicolumn{2}{|c|}{ Interaction totale } \\
\hline $\begin{array}{c}(1 \mathrm{~d} \text { d.1. }) \\
25,30^{* * *}\end{array}$ & \multicolumn{2}{|c|}{$\begin{array}{c}(1) \\
0,00\end{array}$} & \multicolumn{2}{|c|}{$\begin{array}{c}(1) \\
205,30^{* * *}\end{array}$} & \multicolumn{2}{|c|}{$\begin{array}{c}(4) \\
2,22\end{array}$} \\
\hline
\end{tabular}

Nombre de d.1. de la variance résiduelle : 413.

$*$,**, ****: respectivement $\mathbf{P}<0,05 ; \mathbf{P}<0,01 ; \mathbf{P}<0,001$.

Concernant les différences entre génotypes au locus $N a$, les tableaux 2,3 et 4 font apparaître des résultats convergents. Qu'il s'agisse de l'ensemble des 2 sexes de la première éclosion ou des mâles des 2 lots réunis, les animaux cou $n u$ diffèrent de leurs homologues normalement emplumés de façon significative ou hautement significative dans le sens suivant : ils ont un plumage relativement réduit, la région du cou après plumaison l'est également; le pourcentage des viscères et du cœur par rapport à leur poids vif est plus élevé, ainsi que le pourcentage de l'ensemble de la carcasse éviscérée, ceci essentiellement à cause de la réduction du plumage; enfin leur pourcentage de viande de la région thoracique est supérieur. En outre, le pourcentage correspondant par rapport à la carcasse éviscérée est également significativement plus élevé dans le génotype $\mathrm{Na} n \mathrm{a}^{+}$si l'on considère les mâles des 2 éclosions réunis (tabl. 4), et pour les 2 sexes réunis dans la première éclosion la différence est de même sens, sans atteindre le seuil de 5 p. 100 de signification $(\mathrm{P}<0,10)$. Enfin, également pour les mâles des 2 éclosions réunies, le pourcentage de peau et de graisse intermusculaire de la région thoracique est le plus faible pour le génotype cou nu hétérozygote. 


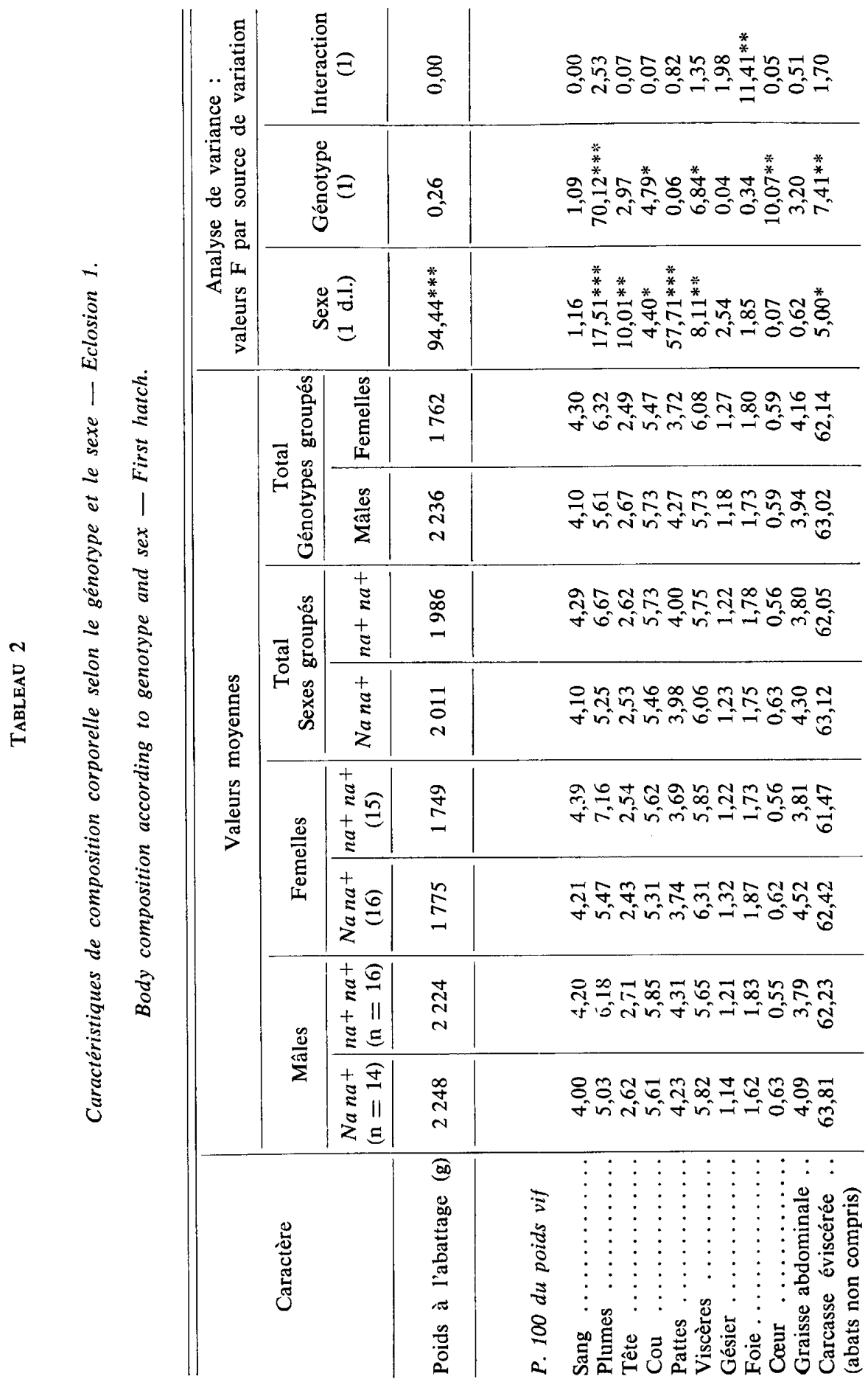




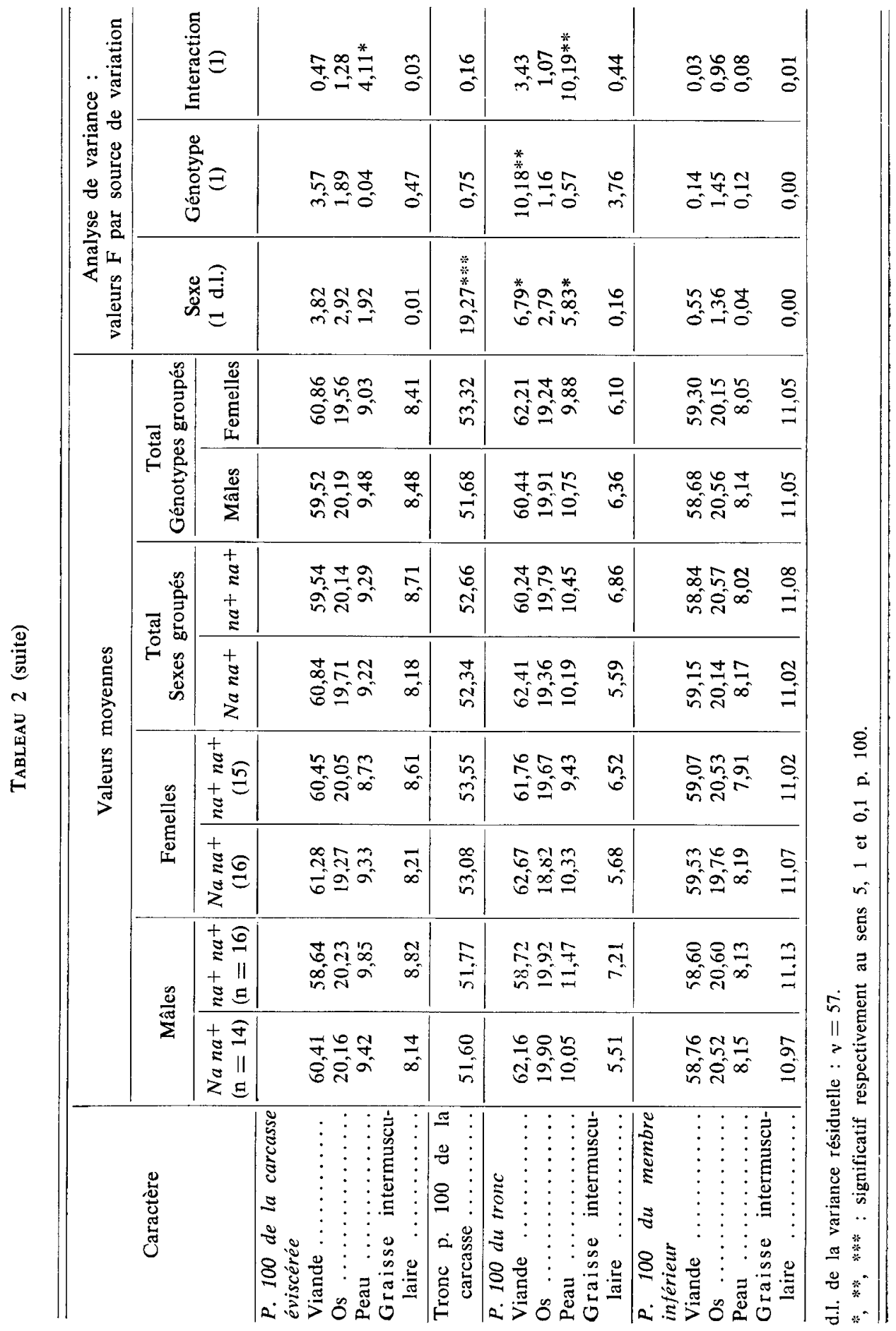




\section{Tableau 3}

Caractéristiques de composition corporelle selon le génotype - Mâles, éclosion 2.

Body composition according to genotype - Males, second hatch.

\begin{tabular}{|c|c|c|c|}
\hline \multirow[b]{2}{*}{ Caractère } & \multicolumn{2}{|c|}{ Valeurs moyennes } & \multirow{2}{*}{$\begin{array}{c}\text { Analyse de variance : } \\
\text { valeur } F \\
\text { (effet Génotype) }\end{array}$} \\
\hline & $\begin{array}{l}N a n a+ \\
(\mathrm{n}=18)\end{array}$ & $\begin{array}{c}n a n a+ \\
(\mathrm{n}=18)\end{array}$ & \\
\hline Poids à l'abattage $(g) \ldots \ldots \ldots \ldots \ldots$ & 2087 & 2029 & 0,64 \\
\hline \multicolumn{4}{|l|}{ P. 100 du poids vif } \\
\hline 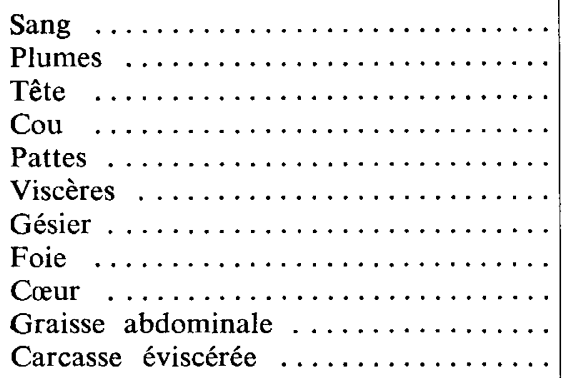 & $\begin{array}{r}3,75 \\
5,58 \\
2,57 \\
5,57 \\
4,33 \\
7,52 \\
1,25 \\
2,10 \\
0,59 \\
3,26 \\
61,85\end{array}$ & $\begin{array}{r}3,75 \\
7,33 \\
2,68 \\
6,21 \\
4,20 \\
6,73 \\
1,26 \\
2,04 \\
0,57 \\
3,02 \\
60,11\end{array}$ & $\begin{array}{l}0,00 \\
63,30^{* * *} \\
2,47 \\
17,11^{* * *} \\
1,14 \\
10,07 * * \\
0,02 \\
1,42 \\
1,13 \\
0,18 \\
13,47^{* * *}\end{array}$ \\
\hline \multicolumn{4}{|l|}{ P. 100 de la carcasse éviscérée } \\
\hline 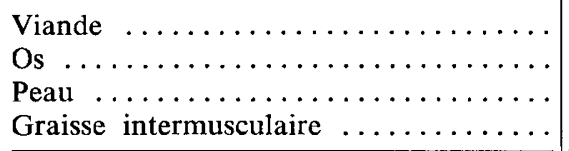 & $\begin{array}{r}62,22 \\
20,16 \\
8,14 \\
7,98\end{array}$ & $\begin{array}{r}60,80 \\
20,52 \\
8,60 \\
7,83 \\
\end{array}$ & $\begin{array}{l}2,88 \\
0,61 \\
2,57 \\
0,03\end{array}$ \\
\hline Tronc p. 100 de la carcasse $\ldots \ldots \ldots$ & 52,36 & 52,01 & 0,68 \\
\hline \multicolumn{4}{|l|}{ P. 100 du tronc } \\
\hline 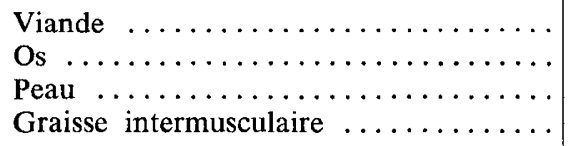 & $\begin{array}{r}63,55 \\
19,71 \\
9,56 \\
4,83\end{array}$ & $\begin{array}{r}61,72 \\
20,10 \\
10,20 \\
5,34\end{array}$ & $\begin{array}{l}4,08\left(^{*}\right) \\
0,59 \\
1,73 \\
0,61\end{array}$ \\
\hline \multicolumn{4}{|l|}{ P. 100 du membre inférieur } \\
\hline 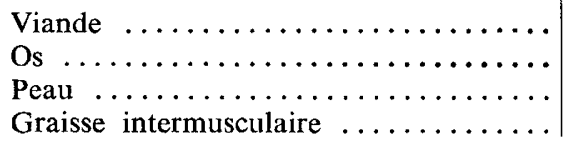 & $\begin{array}{r}61,06 \\
20,77 \\
6,62 \\
10,54\end{array}$ & $\begin{array}{r}59,87 \\
21,01 \\
6,88 \\
10,50\end{array}$ & $\begin{array}{l}1,44 \\
0,17 \\
0,73 \\
1,00\end{array}$ \\
\hline
\end{tabular}

$(*),{ }^{* *}, *^{* * *}:$ respectivement $\mathbf{P}<0,10 ; \mathbf{P}<0,05 ; \mathbf{P}<0,01 ;$ $\mathbf{P}<0,001$. 


\section{Tableau 4}

Analyse de variance des effets "génotype» et «éclosion》sur les mâles.

Variance analysis of the «genotype 》 and «hatch» effects on males.

\begin{tabular}{|c|c|c|c|}
\hline \multirow[b]{2}{*}{ Caractère } & \multicolumn{3}{|c|}{ Valeur de $F$ par source de variation } \\
\hline & $\begin{array}{l}\text { Eclosion } \\
\text { (1 d.1.) }\end{array}$ & $\begin{array}{l}\text { Génotype } \\
\text { (1) }\end{array}$ & $\begin{array}{c}\text { Interaction } \\
\text { (1) }\end{array}$ \\
\hline Poids à l'abattage $(g) \ldots \ldots \ldots \ldots \ldots$ & $10,19 * *$ & 0,57 & 0,12 \\
\hline P. 100 du poids vif & & & \\
\hline 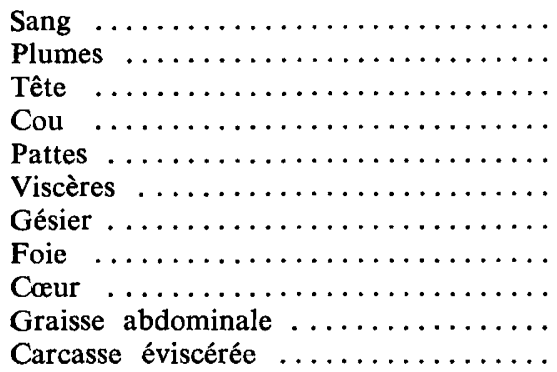 & $\begin{array}{l}5,37^{*} \\
23,80^{* * *} \\
0,51 \\
1,77 \\
0,00 \\
83,14^{* * *} \\
2,11 \\
60,93^{* * *} \\
0,31 \\
8,42^{* *} \\
31,13^{* * *}\end{array}$ & $\begin{array}{l}0,44 \\
69,09^{* * *} \\
2,96 \\
13,61^{* * *} \\
0,08 \\
10,00^{* *} \\
0,56 \\
2,65 \\
7,79^{* *} \\
0,73 \\
20,61^{* * *}\end{array}$ & $\begin{array}{l}0,44 \\
2,94 \\
0,09 \\
2,80 \\
1,57 \\
4,07 * \\
0,34 \\
9,54^{* *} \\
2,30 \\
0,11 \\
0,05\end{array}$ \\
\hline P. 100 de la carcasse éviscérée & & & \\
\hline 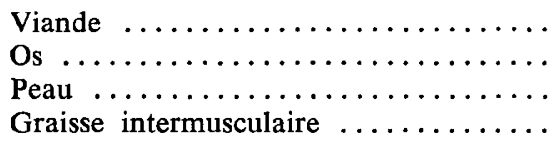 & $\begin{array}{l}9,65^{* *} \\
0,22 \\
17,10^{* *} \\
0,69\end{array}$ & $\begin{array}{l}6,18^{*} \\
0,48 \\
4,84^{*} \\
0,15\end{array}$ & $\begin{array}{l}0,08 \\
0,21 \\
0,26 \\
0,35\end{array}$ \\
\hline Tronc p. 100 de la carcasse $\ldots \ldots \ldots$ & 2,40 & 0,37 & 0,66 \\
\hline P. $100 \mathrm{du}$ tronc & & & \\
\hline 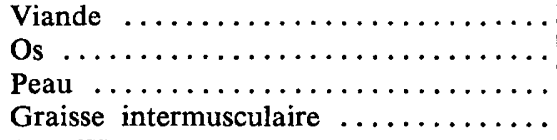 & $\begin{array}{l}11,11^{* * *} \\
0,00 \\
5,96^{*} \\
5,37^{*}\end{array}$ & $\begin{array}{l}15,91 * * * \\
0,25 \\
8,19^{* *} \\
4,05^{*}\end{array}$ & $\begin{array}{l}1,47 \\
0,21 \\
1,17 \\
1,17\end{array}$ \\
\hline P. 100 du membre inférieur & & & \\
\hline 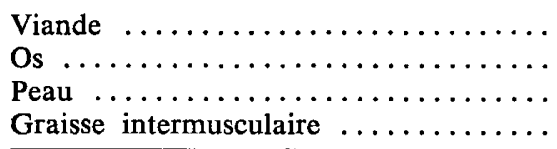 & $\begin{array}{l}5,24^{*} \\
0,86 \\
19,21^{* * *} \\
0,37\end{array}$ & $\begin{array}{l}0,76 \\
0,20 \\
0,14 \\
0,01\end{array}$ & $\begin{array}{l}0,44 \\
0,05 \\
0,20 \\
0,01\end{array}$ \\
\hline
\end{tabular}

*, **, *** : respectivement $\mathrm{P}<0,05 ; \mathrm{P}<0,01 ; \mathrm{P}<0,001$. 
L'avantage de rendrement associé à ce génotype, tant par la moindre quantité de plumage que par la plus grande importance relative de la musculature dans la carcasse éviscérée, était hautement significatif dans les résultats de ZEIN-EL-DeIN et al. (1984 b). En pourcentage, l'importance des différences correspondantes est moindre dans le cas présent que dans ceux cités : en tenant compte du total des animaux disséqués dans les 2 sexes et les 2 éclosions pour les mâles, le gain associé au gène $N a$ sur le rendement en viande, en pourcentage du poids vif, est ici légèrement inférieur à 2,5 p. 100 . Cela représente cependant entre 3,5 et 4 p. 100 de viande obtenue en plus pour le génotype cou nu comparé au génotype nat nat à partir d'un même poids vif. On peut remarquer, sans pouvoir en tirer une conclusion, que l'avantage associé au gène $N a$ est plus grand en valeur relative dans des populations à potentiel de croissance moins élevé.

Les résultats de HANZL \& Somes (1983), comme le présent travail et ceux déjà cités, font apparaître une supériorité des poulets cou nu pour le rendement de la carcasse apprêtée et éviscérée. Ces auteurs notent aussi une teneur inférieure en lipides de la carcasse totale pour les génotypes $\mathrm{NaNa}$ et $\mathrm{Na} \mathrm{na+}$, ce qui concorde avec la différence trouvée pour le gras intermusculaire chez les mâles dans le présent travail et dans les 2 sexes par ZEIN-EL-DeIN et al. (1984 b).

Enfin la précision apportée par notre travail, selon laquelle la proportion plus élevée de tissu musculaire se localise dans la partie thoracique peut s'accorder avec les résultats de Somes \& JoHNSON (1982), selon lesquels une meilleure conformation de la poitrine est assuciée au gène $s c$, d'effet analogue à $N a$ mais plus extrême quant à la réduction du plumage.

Nos résultats vérifient donc que, dans un croisement se rapprochant du type poulet de chair, le gène cou nu n'a aucun effet défavorable sur la croissance à température modérée et améliore légèrement mais de façon non négligeable le rendement à l'abattage et en viande. D'autres résultats nous ayant montré qu'avec un chauffage prolongé après 4 semaines, soit à $31^{\circ} \mathrm{C}$ (MonNet et al., 1979) soit à $29^{\circ} \mathrm{C}$ pour un groupe en cages et $24^{\circ} \mathrm{C}$ pour des femelles au sol (ZEIN-EL-DEIN et al., 1984 a), l'indice de consommation des oiseaux cou nu n'était pas détérioré par rapport aux animaux normalement emplumés, il sera intéressant de comparer le gain de rendement prévisible avec le coût supplémentaire résultant d'un chauffage modéré prolongé jusqu'à l'abattage.

Reçu le 14 décembre 1984.

Accepté le 6 mars 1985.

\section{Remerciements}

Nous remercions M. DA DaLt, Directeur de l'Ecole Nationale d'Aviculture, Rambouillet, d'avoir assuré l'élevage et les mesures de croissance des animaux utilisés dans cette expérience. 


\section{Références bibliographiques}

Bordas A., Merat P., Sergent D., Ricard F., 1978. Influence du gène $\mathrm{Na}$ (cou nu) sur la croissance, la consommation alimentaire et la composition corporelle du poulet selon la température ambiante. Ann. Génét. Sél. Anim., 10, 209-231.

HaNZl C.J., Somes R.G., 1983. The effect of the Naked Neck gene $N a$ on growth and carcass composition of broilers raised in two temperatures. Poultry Sci., 62, 934-941.

Monnet L.E., Bordas A., Mérat P., 1979. Gène cou nu et performances de croissance selon la température chez le poulet. Ann. Génét. Sél. Anim., 11, 397-412.

Ricard F.H., Rouvier R., 1967. Etude de la composition anatomique du poulet de chair. I. Variabilité de la répartition des différentes parties corporelles chez des coquelets Bressepile. Ann. Zootech., 16, 23-29.

SOMES R.G., Johnson Susan, 1982. The effect of the scaleless gene, $s c$, on growth performance and carcass composition of broilers. Poultry Sci., 61, 414-423.

Zein-El-Dein A., Zeiny M., Ayour H., 1981. Carcass measurements on Naked Neck and normal chicks. Ann. Genet. Sel. Anim., 13, 435-440.

Zein-El-Dein A., Bordas A., Mérat P., 1984 a. Croissance et efficacité alimentaire de poulets cou nu ou normalement emplumés selon la teneur en protéines de la ration. Génét. Sél. Evol., 16, 355-366.

Zein-El-Dein A., Bordas A., Mérat P., 1984 b. Composition corporelle de poulets cou nu ou normalement emplumés selon le taux protéinique de la ration. Génét. Sél. Evol., 16, 491-502. 